\title{
Originales
}

\section{Análisis del absentismo por incapacidad temporal en personal universitario}

\section{Analysis of employee absenteeism caused by temporary disability in university staff}

\section{M. 'Avelina Rubio-Garlito', Eloísa Julia Guerrero-Barona² y Antonio José Moreno-Gómez'}

1. Servicio de Prevención. Universidad de Extremadura. Cáceres. España.

2. Departamento de Psicología y Antropología. Facultad de Educación. Universidad de Extremadura. Badajoz. España.

\section{Recibido: 23-10-15}

Aceptado: 15-12-15

\author{
Correspondencia \\ M. ${ }^{a}$ Avelina Rubio Garlito \\ Servicio de Prevención. Edificio de Usos Múltiples. \\ Campus Universitario. Universidad de Extremadura, 10071 Cáceres. España. \\ Teléfono: 676813756 \\ Correo electrónico: arubiogar@unex.es
}

Resumen

Objetivos: Describir el absentismo laboral por Incapacidad Temporal (IT) y analizar la evolución del Índice de Absentismo Absoluto en la Universidad de Extremadura (UEx), con el fin de valorar la posible influencia que la nueva reglamentación de la IT a partir del año 2012 pueda tener en esa evolución.

Métodos: estudio exploratorio de corte epidemiológico (prevalencia) de 2055 procesos de IT registrados durante el periodo 2009-2013, en el que se describe la frecuencia y duración de las bajas en relación a variables demográficas y laborales. La evolución del Índice de Absentismo Absoluto se determinó mediante el cálculo del mismo en cada año del periodo de estudio.

Resultados: La mayor frecuencia de IT por edad se registra entre los 50 y 64 años; el 57,8\% de los procesos de IT corresponden a mujeres y el 42,2\% a hombres. El Personal de Administración y Servicios (PAS) registra el 52\% de los procesos de IT y el Personal Docente Investigador (PDI) el 48\%. En cuanto a la evolución del índice de absentismo, se observa un aumento progresivo entre 2009-2012 y un descenso considerable en 2013.

Conclusiones: Existe un aumento de la IT con la edad, las mujeres registran más bajas que los hombres y el PAS más que el PDI. El descenso del índice de absentismo en 2013 podría deberse a los efectos del Real Decreto-ley 20/2012, pues ante la perspectiva de ver reducido su salario, los trabajadores de la UEx podrían decidir asistir al trabajo, aún sin estar en óptimas condiciones (presentismo laboral).

Med Segur Trab (Internet) 2015; 61 (241) 436-447

Palabras clave: Absentismo, estudio epidemiológico, incapacidad temporal, presentismo.

Abstract

Objetives: Describe the work absenteeism for temporary disability; the evolution of Absolute Absenteeism Rate is also analyzed, taking into account the potential influence of the new 2012 temporary disability regulation.

Methods: 2055 processes of temporary disability recorded during 2009-2013 which are analyzed by means of an epidemiological exploratory study (prevalence). It describes off-work frequency and duration 
as related to demographic and labor variables. The evolution of Absolute Absenteeism Rate was determined by calculating the same in each year of the study period.

Results: The higher frequency of temporary disability is recorded between 50 and 64 years; $57.8 \%$ of the processes are women and $42.2 \%$ men. The personnel of administration and services record $52 \%$ of the processes and professors and researchers $48 \%$.In terms of the employee absenteeism rate, a progressive rise occurs during 2009-2012, decreasing considerably in 2013.

Conclusions: There is an increase in the temporary disability due to factors such as age, that women employees present a higher rate of absences than men and that the personnel of administration and services present a higher rate of absences than professors and researchers. The descent of the employee absenteeism rate in 2013 might be due to the effects of the Royal Decree-Law 20/2012, so that UEx employees would go to work, even without being in optimal conditions (Presenteeism), as to avoid a reduction in their salary.

Med Segur Trab (Internet) 2015; 61 (241) 436-447

Keywords: Absenteeism, epidemiological study, temporary disability, presenteeism. 


\section{INTRODUCCIÓN}

El absentismo laboral es percibido en la actualidad como un problema relevante. El interés por el tema es compartido por los diferentes agentes implicados (gobierno, empresarios, mutuas y sindicatos) quienes debaten sobre la necesidad de regularlo, controlarlo y reducirlo debido a los elevados costes económicos, sanitarios y sociales que genera.

La mayoría de los investigadores que analizan el absentismo laboral señalan que es un fenómeno complejo y difícil de abordar. La complejidad viene determinada por la diversidad de conductas de ausencia que se engloban en el término absentismo. La dificultad para su análisis radica, por una parte, en la inexistencia de una definición delimitada y concreta del fenómeno («la definición de absentismo suele ser poco más que lo que se puede medir en cada base de datos ") ${ }^{1}$; por otra, porque no existen fuentes estadísticas oficiales que tengan alcance nacional y cuyos datos sean homogéneos ${ }^{2}$. Por último, porque la utilización de diferentes índices para su cuantificación dificulta la realización de estudios comparativos, ya que no siempre se computan el mismo tipo de ausencias $^{2}$ y los índices de absentismo utilizados habitualmente representan magnitudes diferentes al haber sido elaborados con sistemas de cálculo diferentes ${ }^{3}$.

De lo anteriormente expuesto se deduce la necesidad de unificar criterios tanto para su contextualización como para su cuantificación. Fernández y Herrero señalan que, «se hace preciso la aprobación de un sistema comunitario homologado de definición de parámetros básicos y de indicadores de absentismo»" Igualmente, en el Seminario sobre la Gestión del absentismo laboral y la incapacidad temporal en Europa (Madrid, junio de 2011), organizado por CISAL (Centro de Investigación en Salud Laboral), se puso de manifiesto que para hablar de absentismo es necesario homogeneizar criterios y consolidar datos, ya que se trata de un fenómeno complejo que requiere una visión integral ${ }^{5}$.

A la hora de analizar el absentismo laboral, los investigadores señalan la existencia de una serie de variables o factores íntimamente relacionados con el mismo que inciden en la frecuencia y duración de las bajas y que tienen un efecto directo, indirecto o moderador sobre los niveles de absentismo ${ }^{6}$. Entre estas variables se encuentran el sexo, la edad, la ocupación, el tipo de contrato y las condiciones de trabajo ${ }^{7}$. Igualmente, los riesgos laborales en general, el mobbing, la tensión en el trabajo y la cultura corporativa son antecedentes del absentismo incidiendo de forma significativa en el mismo ${ }^{8}$.

Dentro de las diferentes conductas de ausencia que engloba el absentismo laboral (previsto y justificado, injustificado, etc.), las ausencias por motivos de salud, la IT, son la causa más importante de la no asistencia al trabajo. Así, la Fundación Europea para la Mejora de las Condiciones de Vida y de Trabajo, en su informe La prevención del absentismo en el lugar de trabajo, señala que la principal causa que mantiene a los trabajadores alejados de sus puestos de trabajo es la enfermedad ${ }^{9}$. En la misma línea, Peiró et al ${ }^{10}$ afirman que los problemas de salud causan la mayoría de las horas perdidas en el trabajo, y Sánchez Gallo ${ }^{11}$ apunta que las contingencias comunes y profesionales son la principal causa de absentismo de larga duración, mientras que los factores psicosociales están más relacionados con el de corta duración.

En la mayoría de los estudios sobre el absentismo laboral, sea cual sea el enfoque desde el que se analice, se evidencia la necesidad de profundizar en su conocimiento no sólo como estrategia de choque y control para la reducción de su coste, sino "como un indicador de salud organizacional, que ha de ser tratado desde un punto de vista integrador de la organización en el que además de desarrollar políticas preventivas de riesgos laborales y mejora de las condiciones de trabajo, se potencie el bienestar del trabajador como vía para la consecución de la excelencia organizacional» ${ }^{6}$.

Desde el enfoque de la salud laboral este trabajo se plantea como una primera aproximación al conocimiento del absentismo laboral en la Universidad de Extremadura (UEx), mediante la descripción del perfil de las ausencias laborales por motivos de 
salud (IT) en el periodo 2009-2013. Concretamente, se pretende conocer la influencia tanto de determinadas variables, demográficas y laborales, como el efecto en los comportamientos de ausencia de la nueva reglamentación de la IT del año 2012. Este estudio preliminar puede ser la base para, posteriormente, realizar un análisis más profundo de sus causas que permita establecer programas de intervención como medida preventiva y de control del absentismo laboral.

\section{MATERIAL Y MÉTODOS}

Se trata de un estudio epidemiológico en el que se analiza la prevalencia de la IT y la evolución del índice de absentismo en el periodo 2009-2013.

\section{Población de estudio}

La muestra está compuesta por los procesos de IT registrados durante el periodo de estudio, concretamente se analizan 2055 procesos. Por lo tanto, la unidad muestral es el proceso de ausencia por enfermedad, no el trabajador. La distribución anual de los procesos de IT, así como el total de trabajadores y su distribución por sexo y colectivo se refleja en la tabla I.

Tabla I. Total de trabajadores de la Universidad de Extremadura distribuidos por colectivo y género y frecuencia de procesos de Incapacidad Temporal (Años 2009-2013)

\begin{tabular}{|c|c|c|c|c|c|c|c|c|c|}
\hline \multirow{3}{*}{ AÑO } & \multicolumn{6}{|c|}{ COLECTIVO } & \multirow{3}{*}{$\begin{array}{c}\text { Total } \\
\text { trabajadores }\end{array}$} & \multirow{3}{*}{$\begin{array}{l}\text { Fecuencia } \\
\text { de procesos }\end{array}$} & \multirow{3}{*}{ Porcentaje } \\
\hline & \multicolumn{3}{|c|}{$\begin{array}{c}\text { Personal de Administración } \\
\text { y Servicios }\end{array}$} & \multicolumn{3}{|c|}{$\begin{array}{l}\text { Personal Docente } \\
\text { Investigador }\end{array}$} & & & \\
\hline & TOTAL & Mujeres & Hombres & TOTAL & Mujeres & Hombres & & & \\
\hline 2009 & 848 & 452 & 396 & 1.940 & 694 & 1.246 & 2.788 & 456 & 22,2 \\
\hline 2010 & 859 & 459 & 400 & 1.990 & 709 & 1.281 & 2.849 & 420 & 20,4 \\
\hline 2011 & 853 & 456 & 397 & 1.869 & 665 & 1.204 & 2.722 & 438 & 21,3 \\
\hline 2012 & 860 & 458 & 402 & 1.827 & 651 & 1.176 & 2.687 & 435 & 21,2 \\
\hline 2013 & 859 & 457 & 402 & 1.864 & 682 & 1.182 & 2.723 & 306 & 14,9 \\
\hline TOTAL & & & & & & & & 2.055 & 100,0 \\
\hline
\end{tabular}

\section{Instrumentos}

Los indicadores de absentismo empleados son la prevalencia o frecuencia de procesos de IT durante los años 2009-2013, la duración media de los procesos de IT y el Índice de Absentismo Absoluto o Tasa Global de Ausencia. Este índice se define como el cociente entre los días de ausencia de todos los trabajadores y el total de días de trabajo teórico previsto multiplicado por 100.

El análisis estadístico de los datos, una vez codificados en formato Hoja de cálculo Microsoft Excel 2007, se llevó a cabo con IBM SPSS Statistics v 19, mediante el cual se realizó un análisis descriptivo de las variables y su distribución de frecuencias (absoluta y relativa) y se analizaron las asociaciones bivariantes de la edad y la duración de los procesos de IT, en cada una de las variables contempladas en el estudio.

\section{Procedimiento}

Las fuentes de información utilizadas en el desarrollo de este estudio han sido:

- Aplicaciones Universitas XXI. Apartado UXXI-RRHH (Explotación): base de datos que recoge anualmente las ausencias laborales debidas a IT. 
- FileMaker Pro10: base de datos del Servicio de Prevención de la UEx de la que se obtienen los datos referidos a las variables de estudio (demográficas y laborales).

- Estadísticas universitarias elaboradas por la Unidad Técnica de Evaluación y Calidad de la UEx, de las que se han obtenido datos totales de número de trabajadores para los años comprendidos en el estudio.

A partir de los datos disponibles, por un lado se realizó un estudio exploratorio de corte epidemiológico y transversal en el que se analizó la prevalencia de las ausencias laborales debidas a IT, describiendo la frecuencia y duración de las bajas en relación a las variables establecidas:

- Variables demográficas: edad y género.

- Variables laborales: colectivo al que pertenecen los trabajadores, Personal Docente Investigador (PDI) y Personal de Administración y Servicios (PAS) y categoría profesional de los mismos.

Para establecer las categorías profesionales se han tenido en cuenta los siguientes aspectos:

- Personal Docente e Investigador: Se han utilizado las categorías profesionales que aparecen en la Ley Orgánica 6/2001, de 21 de diciembre, de Universidades ${ }^{12}$.

- Personal de Administración y Servicios: Dada la cantidad de categorías y especialidades de este colectivo se ha optado por agruparlas de acuerdo a la afinidad de funciones.

Por otro lado, mediante el cálculo del Índice de Absentismo Absoluto, se analiza la evolución del mismo a lo largo del periodo de estudio, considerando el posible efecto de la crisis económica y de la nueva regulación de la IT a partir de 2012 (cambios legislativos) en esta evolución.

\section{RESULTADOS}

En primer lugar, al tratarse de un estudio epidemiológico, se exponen los resultados del análisis de la frecuencia de la IT en cada una de las variables cualitativas (género, colectivo y categoría profesional,) en relación a las variables cuantitativas (edad de los trabajadores y duración de las bajas). En segundo lugar, se analiza la evolución del índice de absentismo en los años 2009-2013.

\section{Variables demográficas y frecuencia de la IT}

\subsection{Descripción de la IT por edad y duración de las bajas}

La edad media de los empleados de la UEx que a lo largo de los cinco años del periodo de estudio han presentado, al menos, un proceso de IT es de 47,20 años $(D T=9,56)$. El rango de edad está comprendido entre los 21 y los 68 años.

La distribución de los datos refleja un aumento de la frecuencia de la IT desde los 21 hasta los 48 años de edad, donde aparece el pico más elevado, a partir del cual empieza a decrecer paulatinamente hasta los 58-60 años, en los que se observa una nueva subida. A partir de esta edad hay un decrecimiento hasta los 68 años, más acusado a los 65 años, coincidiendo con la edad de jubilación.

Así mismo, se observa que la frecuencia de los procesos de IT es mayor entre los 50 y 64 años que entre los 21 y 46 años, lo que pone de manifiesto que hay un aumento de la frecuencia de la IT con la edad.

En cuanto a la duración de las bajas, la media de los días de baja, a lo largo del periodo de estudio, por cada proceso de IT es de 42,90 $(D T=67,96)$ con un rango con valores comprendidos entre 1 y 546 días, siendo el total de días de baja para todo el 
periodo de 87761 días, cuyo desglose anual se refleja en la figura 1. La mayor frecuencia de procesos de IT tiene una duración entre 1 y 15 días.

Figura 1. Total de días de baja por Incapacidad Temporal (2009-2013)

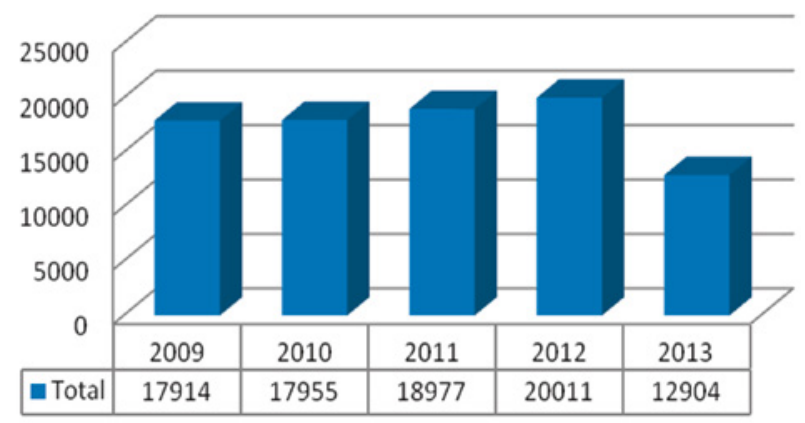

\subsection{Descripción de la IT por género, edad y número de días de baja}

Durante el periodo 2009-2013 la distribución por género del total de trabajadores de la UEx, presenta un mayor porcentaje de hombres que de mujeres, como se observa en la figura 2. Globalmente, en dicho periodo, la media de mujeres es de $1137(41,29 \%)$ y la de hombres es de 1617 (58,71\%).

Figura 2. Distribución de los trabajadores de la Universidad de Extremadura por género (2009-2013)

$$
\text { " MUJER = HOMBRE }
$$

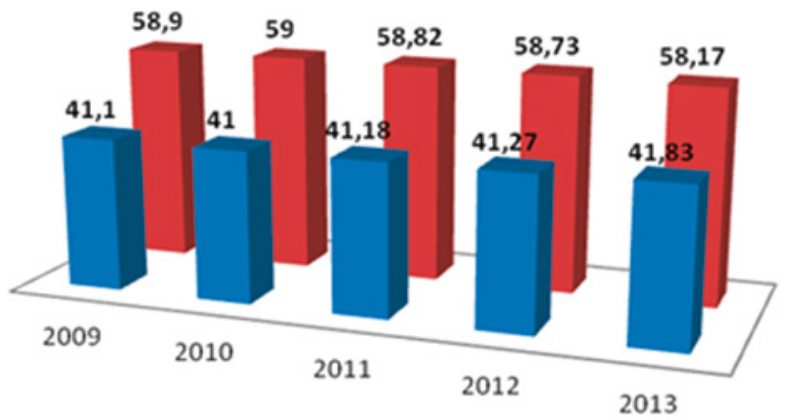

Por lo que respecta a las bajas laborales, el 57,8\% (1187) de los procesos de IT corresponden a mujeres y el 42,2\% ( 868) corresponden a hombres.

La edad media de las mujeres que inician un proceso de IT es de 45,16 años $(D T=9,10)$ y la de los hombres es de 50,06 años $(D T=9,47)$.

La mayor frecuencia de procesos de baja por IT se observa alrededor de los 48 años en ambos géneros. En las mujeres se observa una mayor distribución de los procesos de los 21 a los 48 años que de estos a los 65 años. Las frecuencias son similares en ambos periodos. En cuanto a los hombres, a partir de los 48 hasta los 64 años las frecuencias de IT son mucho más elevadas que de los 25 a los 48 años, observándose incrementos importantes de las mismas a los 51 años y de los 59 a los 61 años.

En cuanto a la duración media de los procesos de IT en la mujer es de 44,23 $(D T=69,96)$ días y en el hombre de 41,09 $(D T=65,14)$ días. La mayor frecuencia de procesos de IT se presenta entre 1 y 16 días de duración de la baja en el caso de la mujer y entre 1 y 32 días en el caso del hombre. 


\section{Variables laborales y frecuencia de la IT}

\subsection{Descripción de la IT por colectivo (PAS/PDI), edad y duración de las bajas}

Globalmente, a lo largo del periodo de estudio, la UEx cuenta con una media de 2754 trabajadores, de los cuales el 31,08\% (856) pertenecen al PAS y el 68,92\% (1898) pertenecen al PDI.

En relación a las bajas laborales, el 52\% (1069) de los procesos de IT corresponden al PAS mientras que el $48 \%$ (986) corresponden al PDI.

En cuanto a la edad media en el inicio de un proceso de IT, se observan pocas diferencias entre ambos colectivos $(M=46,68, D T=9,37$ en el PAS y $M=47,79, D T=9,75$ en el PDI). En el PAS el rango de edad oscila entre 22 y 64 años mientras que en el PDI oscila entre 21 y 68 años. En ambos colectivos el mayor número de bajas se produce a los 48 años. Las frecuencias más elevadas en el PAS ocurren entre los 43-59 años y en el PDI entre los 36-63 años.

La duración media de la IT en el PAS es de 44,60 $(D T=78,14)$ días, con un rango entre 1 y 546 días de duración del proceso. En el PDI la duración media es de 41,05 $(D T=54,86)$ días y un rango de duración de la baja entre 1 y 455 días.

En cuanto a la duración de los procesos de baja la mayor frecuencia se encuentra entre 1 y 16 días en el PAS y entre 1 y 32 días en el PDI.

\subsection{Descripción de la IT por categoría profesional, edad y duración de las bajas}

La distribución de frecuencias de IT por categoría profesional se refleja en la figura 3, donde se observa que dentro de los dos colectivos a los que está adscrito el personal de la UEx y en cuanto al PDI, la mayor frecuencia de procesos de IT se produce en la categoría de Profesor Titular de Universidad (TU) con un 12,7\% (260), seguida de la categoría de Titular de Escuela Universitaria (TEU) con el 9,9\% (204).Con respecto al PAS la mayor frecuencia de procesos se produce en el Personal Administrativo, 18\% (370) y en el Personal de Conserjería,11\% (226).

Figura 3. Frecuencias de Incapacidad Temporal por categoría profesional (2009-2013)

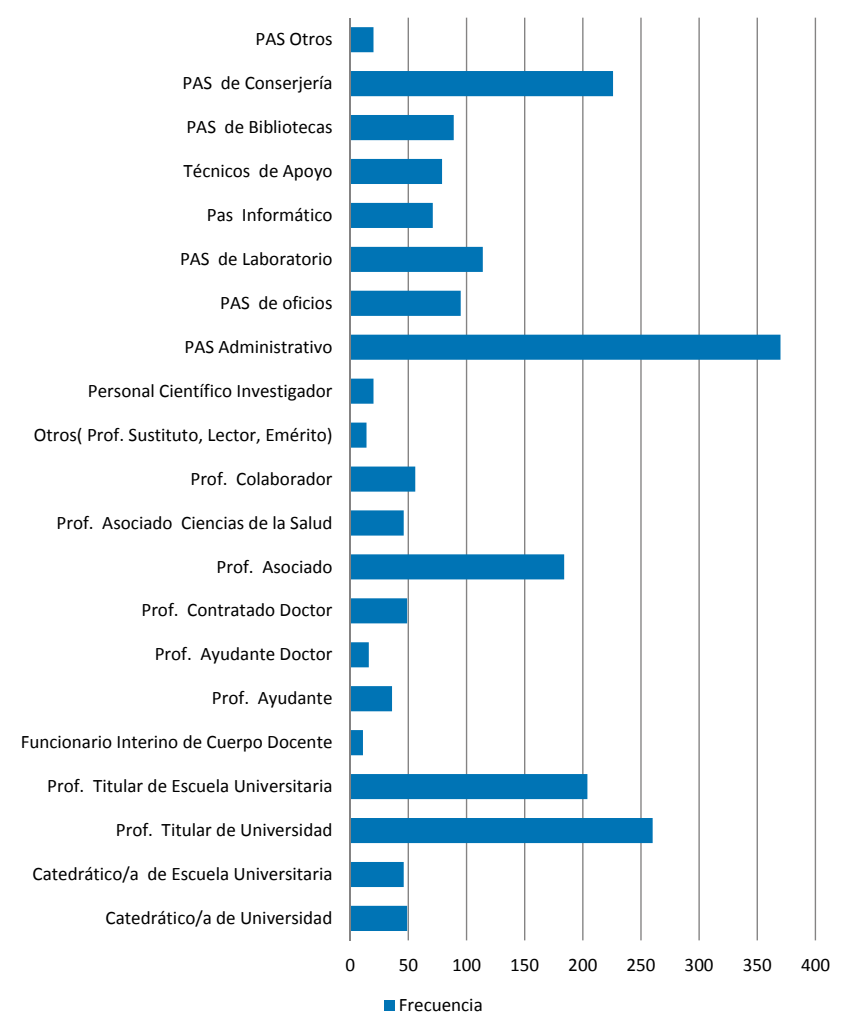

Análisis del absentismo por incapacidad temporal en personal universitario M. ${ }^{a}$ Avelina Rubio-Garlito, Eloísa Julia Guerrero-Barona y Antonio José Moreno-Gómez 
La edad media de los trabajadores que presentan bajas por IT y la media de días de baja por cada categoría se recogen en la tabla II, así como el rango de edad y de días de baja. En las categorías del PAS, la edad media se encuentra entre los 31 y los 50 años y en las categorías del PDI, oscila entre los 30 y los 57 años.

Tabla II. Promedios de edades y número de días de baja de los procesos de Incapacidad Temporal por categoría profesional (2009-2013)

\begin{tabular}{lcccc}
\hline \multicolumn{1}{c}{ Categoría profesional } & Edad media/DT & $\begin{array}{c}\text { Rango* } \\
\text { Años }\end{array}$ & Media días de baja/DT & $\begin{array}{c}\text { Rango* } \\
\text { Días baja }\end{array}$ \\
\hline Catedrático de Universidad & $57,84(5,83)$ & $44-68$ & $37,22(29,54)$ & $3-140$ \\
Catedrático de Escuela Universitaria & $52,93(7,25)$ & $39-67$ & $40,04(25,82)$ & $3-91$ \\
Profesor Titular de Universidad & $47,52(7,36)$ & $34-66$ & $36,10(39,94)$ & $1-455$ \\
Profesor Titular de Escuela Universitaria & $53,00(8,35)$ & $30-66$ & $38,35(47,63)$ & $1-380$ \\
Funcionario Interino de Cuerpos Docentes & $39,36(4,72)$ & $32-44$ & $43,64(62,31)$ & $3-213$ \\
Profesor Ayudante & $34,30(5,73)$ & $27-57$ & $33,94(37,89)$ & $2-150$ \\
Profesor Ayudante Doctor & $39,39(9,65)$ & $30-54$ & $67,44(102,46)$ & $3-388$ \\
Profesor Contratado Doctor & $39,85(6,02)$ & $30-55$ & $48,21(67,70)$ & $2-390$ \\
Profesor Asociado & $48,89(9,25)$ & $21-65$ & $46,98(70,31)$ & $1-395$ \\
Profesor Asociado de Ciencias de la Salud & $51,84(5,82)$ & $42-63$ & $38,50(66,80)$ & $1-389$ \\
Profesor Colaborador & $41,45(7,24)$ & $31-60$ & $54,25(81,77)$ & $2-402$ \\
Otros (Profesor Sustituto, Lector, Emérito) & $35,36(7,86)$ & $28-53$ & $37,38(45,75)$ & $3-179$ \\
Personal Científico Investigador & $30,04(3,40)$ & $25-38$ & $29,79(26,30)$ & $3-94$ \\
PAS Administrativo & $46,73(8,08)$ & $25-63$ & $43,86(81,40)$ & $1-402$ \\
PAS de Oficios & $45,82(10,73)$ & $27-64$ & $44,23(82,85)$ & $1-394$ \\
PAS de Laboratorio & $50,75(6,92)$ & $31-63$ & $52,07(87,66)$ & $1-412$ \\
Pas Informático & $42,85(7,57)$ & $25-63$ & $31,69(54,94)$ & $1-311$ \\
Técnicos de Apoyo & $31,12(6,24)$ & $22-62$ & $24,03(23,88)$ & $1-108$ \\
PAS de Bibliotecas & $49,54(7,90)$ & $24-63$ & $40,85(84,12)$ & $1-546$ \\
PAS de Conserjería & $50,67(7,35)$ & $30-64$ & $56,24(81,70)$ & $1-408$ \\
Otros & $42,21(9,73)$ & $27-60$ & $35,85(71,11)$ & $2-303$ \\
\hline
\end{tabular}

* Mínimo-Máximo.

La mayor frecuencia de procesos de IT, en las cuatro categorías que presentan mayor número de bajas, se encuentran entre los 47-48 años en TU, PAS Administrativo y PAS de Conserjería (de los 48 a los 64 años la frecuencia de procesos de IT es bastante mayor que de los 30 a los 44 años), mientras que en TEU es de 58 a 60 años (con un aumento importante con respecto a las edades anteriores).

Por lo que respecta a la duración de las bajas, el rango de días de baja por IT se encuentra ente 1 y 546, oscilando la duración media de los procesos de IT en las categorías de PDI entre 31 y 67 días y en las categorías de PAS entre 24 y 56 días. En cuanto a las categorías destacadas en cada colectivo, es mayor la duración de las bajas en las de PDI (TU: 1-50 días, TEU: 20-40 días) que en las de PAS (Personal Administrativo: 1-16 días, Personal de Conserjería: 1-20 días).

\section{Evolución del índice de absentismo 2009-2013}

En cuanto a la evolución del Índice de Absentismo se observa (figura 4) un aumento progresivo del mismo desde 2009 hasta 2012 (año que presenta el índice más elevado, 3,4\%) para decaer considerablemente en 2013 (2,2\%). 
Figura 4. Evolución del índice de absentismo en la Universidad de Extremadura (2009-2013)

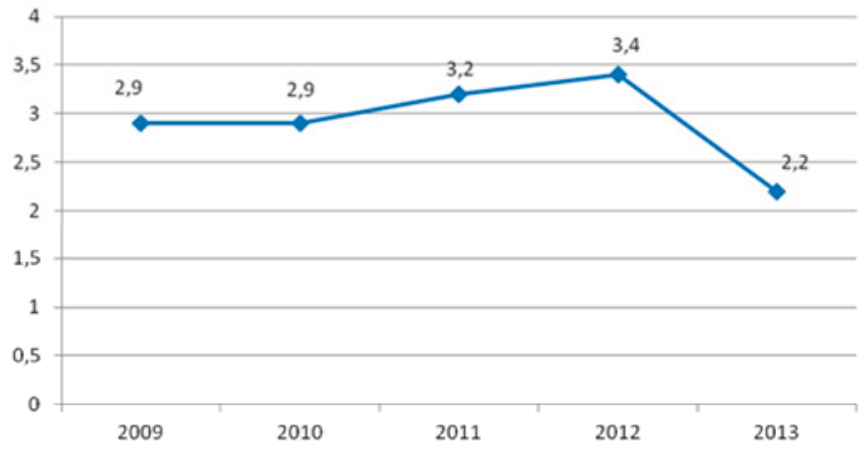

\section{CONCLUSIONES}

El objetivo principal de este trabajo ha sido, por una parte, describir y analizar el perfil de las ausencias laborales por IT en la UEx en el periodo 2009-2013, en relación con las variables demográficas y laborales establecidas, mediante un análisis estadístico descriptivo de los datos obtenidos a partir de distintas fuentes de ámbito universitario; por otra, analizar la evolución del Índice de Absentismo Absoluto durante ese período a fin de comprobar la posible influencia de la nueva reglamentación de la IT en las administraciones públicas a partir de 2012.

Los resultados obtenidos contribuyen a la consecución del objetivo propuesto, permitiendo concluir que existe un aumento de la frecuencia de la IT con la edad en línea con los resultados de algunos estudios sobre absentismo ${ }^{6,13}$. Sin embargo, otros trabajos ${ }^{1,7,10}$ señalan la existencia de un mayor número de procesos de IT entre los trabajadores más jóvenes, siendo la duración media de los procesos mayor a medida que aumenta la edad.

En cuanto a la variable género, las mujeres presentan un mayor porcentaje de procesos de baja por IT que los hombres (siendo menor el número de efectivos a lo largo del periodo de estudio), a una edad media más temprana y con una duración media de los procesos ligeramente más elevada que en los hombres. Resultados similares se ponen de manifiesto en gran parte de la literatura científica sobre absentismo laboral. Algunos autores $^{6,7,13}$ señalan como causas de esta mayor tasa de absentismo en las mujeres las tareas reproductivas y las responsabilidades familiares que estas asumen y sus diferentes condiciones de trabajo, con tareas menos cualificadas, menor posibilidad de promoción y/o salarios más bajos.

Al analizar la influencia de las variables laborales en la frecuencia de IT, se observa que el colectivo del PAS presenta un mayor porcentaje de procesos de baja que el PDI, siendo menor el número de efectivos de este colectivo a lo largo del periodo de estudio. La edad media de inicio de la baja es ligeramente más elevada en el PDI que en el PAS, siendo también más elevado el rango de edad de las bajas. En cuanto a la distribución de frecuencias de IT por edades, en el PDI hay un mayor número de bajas a mayor edad y la duración media de la IT es superior en el PAS que en el PDI.

Por lo que respecta a la categoría profesional de los trabajadores, la mayor frecuencia de procesos, en el periodo de estudio, se produce en dos categorías del colectivo del PAS, Personal Administrativo y Personal de Conserjería, y en dos categorías del colectivo del PDI, Titular de Universidad y Titular de Escuela Universitaria, siendo la edad media de los trabajadores muy similar en las cuatro categorías señaladas.

La mayor frecuencia de procesos de IT se produce en edades similares en las dos categorías del PAS y en la categoría de TU del PDI, mientras que en la categoría de TEU, se produce en edades más avanzadas. 
La duración media de los procesos de IT, es superior en las categorías del PDI (entre 31 y 67 días) que en las categorías del PAS (entre 24 y 56 días).

Por último, al analizar la evolución del índice de absentismo debido a IT se observa un aumento progresivo en los cuatro primeros años del periodo de estudio (2009-2012) para descender considerablemente el último año analizado (2013). Este descenso podría deberse a una disminución de las ausencias laborales como consecuencia de la nueva regulación de la IT en el Real Decreto-ley 20/2012, de 13 de julio, de medidas para garantizar la estabilidad presupuestaria y de fomento de la competitividad ${ }^{14}$. En el mismo se modifican temporalmente las retribuciones durante la situación de IT del personal al servicio de las Administraciones Públicas (tanto el incluido en el Régimen General de la Seguridad Social como el incluido en los regímenes especiales del mutualismo administrativo: MUFACE), limitando la mejora voluntaria de la acción protectora de la Seguridad Social que cada administración pública pudiera establecer.

A partir de su entrada en vigor en la situación de IT, derivada de contingencias comunes, el complemento retributivo que con carácter de mejora voluntaria se puede establecer durante los tres primeros días de baja alcanzará, como máximo, el 50\% de las retribuciones que se vinieran percibiendo en el mes anterior al de causarse la incapacidad. Desde el cuarto hasta el vigésimo día de baja, ambos inclusive, el complemento retributivo que se puede sumar a la prestación económica reconocida por la Seguridad Social o por el mutualismo administrativo no podrá superar el $75 \%$ de las retribuciones. A partir del día vigésimo primero, inclusive, se reconocerá una prestación equivalente al cien por cien de las retribuciones.

Cuando la situación de IT derive de contingencias profesionales, la prestación reconocida podrá ser complementada hasta alcanzar como máximo el 100\% de las retribuciones desde el primer día de la baja.

Las modificaciones contenidas en el Real Decreto-Ley 20/2012, fueron incorporadas a la normativa de la Universidad de Extremadura, mediante la Resolución de 27 de septiembre de 2012, de la Gerencia, por la que se ejecuta el acuerdo adoptado por el Consejo de Gobierno aprobando la normativa para la aplicación y desarrollo en el ámbito de la Universidad de Extremadura de las medidas establecidas para garantizar la estabilidad presupuestaria ${ }^{14}$.

Las modificaciones señaladas anteriormente comenzaron a surtir efecto en los procesos de incapacidad temporal iniciados a partir del 15 de octubre de 2012. Por lo que una posible explicación del descenso del índice de absentismo en 2013 puede ser debido a la nueva reglamentación de la IT. No se puede obviar que la situación de IT conlleva, además de la asistencia sanitaria, una prestación económica que pretende cubrir la falta de ingresos que se produce mientras el trabajador está imposibilitado temporalmente para trabajar. Por lo tanto, cualquier modificación en las condiciones y/o cuantía económica de la prestación puede influir en el comportamiento del trabajador ante una situación de enfermedad. Ante la perspectiva de ver reducido su salario, los trabajadores podrían decidir, en algunos casos, asistir al trabajo sin estar en óptimas condiciones (presentismo) por miedo a la pérdida económica que les supondría.

Entre las limitaciones de este estudio, señalar que el mismo ha tenido que adaptarse a los datos disponibles en las bases de datos universitarias y a la posibilidad de acceso a los mismos. Por ello, entre las diferentes variables que están relacionadas con el absentismo laboral, se han elegido aquellas de las cuales se disponía de datos, no pudiendo analizar otras como salario, tipo de jornada, etc., debido a la falta de información sobre las mismas.

Por último, señalar las limitaciones impuestas por el proceso de análisis estadístico de los datos que, en ocasiones, no ha permitido establecer las correlaciones entre variables deseadas.

No obstante, pese a las limitaciones señaladas, este estudio aporta un primer acercamiento a las características del absentismo laboral en la Universidad de Extremadura que puede servir de base para futuras líneas de investigación en las que, por una parte, 
se analicen los factores que inciden en el absentismo basándose en los modelos psicosociales explicativos del absentismo laboral, cuyos componentes básicos son la autopercepción de salud del trabajador, la capacidad para asistir y la motivación para ausentarse del trabajo y por otra, se aborde su estudio desde una perspectiva de indicador de salud laboral, que permita desarrollar programas de intervención para conseguir, por una parte la reducción del mismo y por otra, como señala Villaplana ${ }^{6}$, mejorar las condiciones de trabajo potenciando el bienestar del trabajador como vía para la consecución de la excelencia organizacional.

En el análisis del absentismo no sólo se deben abordar los factores reactivos de gestión y control, sino también los factores proactivos que aglutinen disciplinas relacionadas tanto con la Prevención de Riesgos Laborales como con la gestión de los Recursos Humanos en las empresas ${ }^{16}$ y su gestión se debe llevar a cabo "cómo un componente más de la cultura preventiva de la empresa ${ }^{6}$.

\section{AGRADECIMIENTOS}

Los autores expresan su agradecimiento al Profesor Carlos Javier Pérez Sánchez del Departamento de Matemáticas de la Universidad de Extremadura, por su ayuda en el análisis estadístico de los datos del estudio.

\section{REFERENCIAS BIBLIOGRÁFICAS}

1. Malo, M.A., Cueto, B., García, C. y Pérez, J.I. La medición del absentismo: Estimaciones desde la perspectiva de las empresas y de las vidas laborales. Disponible en: http://www.seg-social.es/prdi00/ groups/public/documents/binario/174212.pdf. Acceso 14 julio 2014.

2. Porret Gelabert, M. El absentismo laboral en la empresa privada española. Rev. tec. labor.2012; 34(131): 5-81.

3. Ribaya Mallada, F. J. La gestión del absentismo laboral en las empresas españolas. Revista Alta dir. 2008; 43: 29-39.

4. Fernández, M. y Herrero, M. Cuantificación del absentismo laboral en la empresa. Una herramienta para la medición de los niveles de seguridad y salud en la empresa. Prev. trab. salud. 2003; 28: 4-10.

5. Seminario sobre la Gestión del absentismo laboral y la incapacidad temporal en Europa (2011, 22 de junio) Madrid: Ibermutuamur. Disponible en: http://www.ibermutuamur.es/Seminario-sobre-gestion-del.html. Acceso 10 de julio de 2014.

6. Villaplana García, M.D. El absentismo laboral por incapacidad temporal derivada de contingencias comunes en el periodo 2005-2010 [tesis doctoral]. Murcia: Universidad de Murcia; 2012. Disponible en: http://digitum.um.es/xmlui/bitstream/10201/28427/1/M.VILLAPLANA-TESIS\%20ABSENTISMO\%20 LABORAL-2012-pdf.pdf. Acceso 9 de julio de 2014.

7. Benavides, F., Castejón, E. y Murillo, C. La Incapacidad Temporal por Contingencias Comunes en Cataluña. Madrid: Fraternidad Muprespa. Disponible en: http://www.fraternidad.com/descargas/ FM-REVLM-10 7_111_La_incapacidad_temporal_por_contingencias_comunes_en_Cataluna.pdf. Acceso 9 de julio de 2014 .

8. Boada, J., Diego, R. de, Agulló, E. y Mañas, M. A. El absentismo laboral como consecuente de variables organizacionales. Psicothema. 2005; 17 (2):212-218. Disponible en: http://www.psicothema.com/ psicothema.asp?id=3090. Acceso 2 de julio de 2014 .

9. Fundación Europea para la Mejora de las Condiciones de Vida y de Trabajo. La Prevención del Absentismo en el Lugar de trabajo [Resumen]. Luxemburgo: Oficina de Publicaciones Oficiales de las Comunidades Europeas. 1997. Recuperado de: http://www.eurofound.europa.eu/pubdocs/1997/15/es/1/ef9715es.pdf. Acceso 2 de julio de 2014.

10. Peiró, J.M., Rodríguez-Molina, I. y González-Morales, M.G. El absentismo laboral: antecedentes, consecuencias y estrategias de mejora. Valencia: Universitat de Valencia; 2008.

11. Sánchez Gallo, D. El absentismo laboral en España. Del ordenamiento jurídico a la realidad empresarial [tesis doctoral]. Fuenlabrada (Madrid): Universidad Rey Juan Carlos; 2013. Recuperado de: http://hdl. handle.net/10115/11754. Acceso 9 de julio de 2014 .

12. Boletín Oficial del Estado. Ley Orgánica 4/2007, de 12 de abril, por la que se modifica la Ley Orgánica 6/2001, de 21 de diciembre, de Universidades. BOE, núm. 89 de 13/04/ 2007. 
13. Llergo, A. Estudio de la incapacidad temporal en la provincia de Córdoba: factores determinantes y evolución temporal [tesis doctoral].Córdoba: Facultad de Medicina, Universidad de Córdoba; 2003. Disponible en: http://helvia.uco.es/xmlui/bitstream/handle/10396/282/13209668.pdf?sequence=1. Acceso 3 de julio de 2014.

14. Boletín Oficial del Estado. Real Decreto-ley 20/2012, de 13 de julio, de medidas para garantizar la estabilidad presupuestaria y de fomento de la competitividad. BOE, núm. 168 de 14/07/ 2012.

15. Diario Oficial de Extremadura. Resolución de 27 de septiembre de 2012, de la Gerencia, por la que se ejecuta el acuerdo adoptado por el Consejo de Gobierno aprobando la normativa para la aplicación y desarrollo en el ámbito de la Universidad de Extremadura de las medidas establecidas para garantizar la estabilidad presupuestaria. DOE, núm. 189 de 28/09/2012.

16. II Informe Adecco sobre Absentismo: La tasa de absentismo en España se sitúa en el 4,3\%, medio punto porcentual menos que hace un año. [Resumen]. Madrid: Adecco. Disponible en: http://www.adecco.es/_ data/NotasPrensa/pdf/475.pdf. Acceso 28 de junio de 2014.

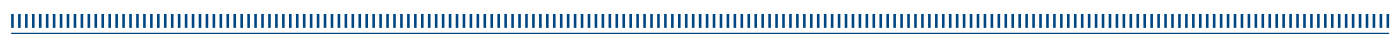

